\title{
Switching of the bacterial flagellar motor near zero load
}

\author{
Junhua Yuan, Karen A. Fahrner, and Howard C. Berg \\ Department of Molecular and Cellular Biology, Harvard University, 16 Divinity Avenue, Cambridge, \\ MA 02138, USA
}

\begin{abstract}
Flagellated bacteria, such as Escherichia coli, are able to swim up gradients of chemical attractants by modulating the direction of rotation of their flagellar motors, which spin alternately clockwise $(\mathrm{CW})$ and counterclockwise $(\mathrm{CCW})$. Chemotactic behavior has been studied under a variety of conditions, mostly at high loads (at large motor torques). Here, we examine motor switching at low loads. Nano-gold spheres of various sizes were attached to hooks (the flexible coupling at the base of the flagellar filament) of cells lacking flagellar filaments in media containing different concentrations of the viscous agent Ficoll. The speeds and directions of rotation of the spheres were measured. Contrary to the case at high loads, motor switching rates increased appreciably with load. Both the $\mathrm{CW} \rightarrow \mathrm{CCW}$ and $\mathrm{CCW} \rightarrow \mathrm{CW}$ switching rates increased linearly with motor torque. Evidently, the switch senses stator-rotor interactions as well as the CheY-P concentration.
\end{abstract}

\section{Keywords}

Escherichia coli; nano-gold; torque; speed; allostery

\section{Introduction}

Cells of Escherichia coli are propelled by several helical flagellar filaments, each driven at its base by a reversible rotary motor. When all the motors on a cell turn counterclockwise (CCW), the filaments coalesce into a helical bundle that pushes the cell steadily forward (in a run). When one or more motors switch to clockwise (CW), their filaments come out of the bundle and go through a series of polymorphic transformations (changes in handedness and shape) that result in uncoordinated movement (a tumble) and choice of a new direction for another run $^{1}$. Tumbles are suppressed when cells move up spatial gradients of chemical attractants ${ }^{2}$. Thus, by controlling the direction of rotation of their flagellar motors, cells are able to move toward regions that are more favorable.

Torque is thought to be generated by electrostatic interactions between the stator protein MotA and the rotor protein FliG, resulting from conformational changes in MotA and MotB (another stator protein), driven by protonation and deprotonation of MotB Asp32 $3 ; 4$. The energy for this process is derived from the change in the electrochemical potential of a proton as it moves from the outside to the inside of the cell. The direction of rotation (switching) is controlled by interaction of the cytoplasmic response regulator CheY-P with the rotor protein FliM $^{5 ; 6 ; 7 . ~}$

Correspondence to: Howard C. Berg.

Publisher's Disclaimer: This is a PDF file of an unedited manuscript that has been accepted for publication. As a service to our customers we are providing this early version of the manuscript. The manuscript will undergo copyediting, typesetting, and review of the resulting proof before it is published in its final citable form. Please note that during the production process errors may be discovered which could affect the content, and all legal disclaimers that apply to the journal pertain. 
Torque generation and switching have been modeled separately: for torque generation, see ${ }^{8}$; 9; for switching, see ${ }^{10 ; 11 ; 12}$.

In earlier work at intermediate and high loads, we found that $\mathrm{CW} \rightarrow \mathrm{CCW}$ switching rates decreased with motor torque, most notably in the speed range between $50 \mathrm{~Hz}$ and stall, while $\mathrm{CCW} \rightarrow \mathrm{CW}$ switching rates remained more nearly constant, with the result that the motor became more CW biased at lower speeds ${ }^{13}$. The highest speeds ( $\sim 200 \mathrm{~Hz}$ at room temperature) were observed with $0.36 \mu \mathrm{m}$ diameter latex beads attached to filament stubs. Recently, we developed an assay that allowed study of the motor near zero load, in which 60-nm diameter gold spheres were attached to hooks of cells lacking flagellar filaments ${ }^{14}$. A dark-field image of such a sphere, observed through a small pinhole, wobbles as the sphere rotates, generating an oscillating signal whose frequency is readily measured ( $\sim 330 \mathrm{~Hz}$ at room temperature). In the present work, by using two orthogonal slits and a second detector, we monitored the direction of rotation as well as the speed. We used gold spheres of different sizes in motility medium containing different concentrations of Ficoll, estimating motor torque from the product of speed times rotational viscous drag coefficient. We found that near zero load both the $\mathrm{CW} \rightarrow \mathrm{CCW}$ and $\mathrm{CCW} \rightarrow \mathrm{CW}$ switching rates increased linearly with motor torque, with motor bias remaining constant. The data could be fit by the model of Duke, LeNovère, \& Bray ${ }^{11}$ in which the activities of a ring of proteins change by conformational spread, assuming that the rates for transitions between active and inactive states of individual subunits are torquedependent.

\section{Results}

Gold spheres coated with anti-hook antibody were attached to hooks of cells stuck to a polylysine-treated glass coverslip. Light scattered from a sphere was focused on two orthogonal slits in front of two photomultiplier tubes, as shown schematically in Fig. 1, generating $x$ and $y$ signals of the sort shown in Fig. 2. These signals, plotted separately (A, B) or in polar coordinates (C), allowed us to measure rotation rates as a function of time (D). Velocity time series were converted into binary time series, from which $\mathrm{CW}$ and $\mathrm{CCW}$ intervals were extracted.

We studied the switching statistics of the motor at near-zero to low loads, using spheres of diameters 100,150 , or $200 \mathrm{~nm}$ in motility medium, or spheres of diameter $200 \mathrm{~nm}$ in motility medium containing Ficoll at 5\%,10\%, or 15\% w/v. The mean CW and CCW intervals were determined for each motor at a specified torque, as shown in Fig. 3. The median and interquartile range of the intervals for each load were calculated and are shown in Fig. $4 \mathrm{~A}$ and $\mathrm{B}$, which include data for intermediate loads from ref. ${ }^{13}$ (for the three lowest loads using 0.36 , 0.54 , and $0.75-\mu \mathrm{m}$ diameter beads on filament stubs in motility medium). Both $\mathrm{CW}$ and $\mathrm{CCW}$ intervals decreased dramatically with load, with the ensemble median decreasing from $\sim 0.8$ to $0.2 \mathrm{~s}$ and from $\sim 4$ to $1 \mathrm{~s}$ for $\mathrm{CW}$ and $\mathrm{CCW}$ intervals respectively. The corresponding switching rates are shown in Fig. $4 \mathrm{C}$ and $\mathrm{D}$, with $\mathrm{k}_{\mathrm{CW} \rightarrow \mathrm{CCW}}=1 /\langle\mathrm{CW}\rangle$ and $\mathrm{k}_{\mathrm{CCW} \rightarrow \mathrm{CW}}=1 /$ $\langle\mathrm{CCW}\rangle$, where $\langle\mathrm{CW}\rangle$ and $\langle\mathrm{CCW}\rangle$ are ensemble medians of $\mathrm{CW}$ and $\mathrm{CCW}$ intervals respectively. Both switching rates increased with motor torque, with a dependence that was approximately linear.

We were concerned that the Ficoll used in our experiments might contain a hydrolysis product (glucose, a chemoattractant) that would perturb the switching statistics, so we did additional measurements with gold spheres of diameter $150 \mathrm{~nm}$ in $5 \%$ Ficoll. In this case, the viscous drag is nearly the same as for spheres of diameter $200 \mathrm{~nm}$ in motility medium. As can be seen in Fig. 5 A, B and C, the CW and CCW intervals and CCW biases were essentially the same. 
The data of Fig. 4 are plotted again in Fig. 6 as a function of CCW speed. The switching rates decreased with speed, as expected, given that motor torque is known to fall linearly in the lowtorque, high-speed regime from a plateau ending at $\sim 160 \mathrm{~Hz}$ to 0 at $\sim 330 \mathrm{~Hz}^{15 ; 16}$. A plot of this part of the torque-speed relationship is shown in Fig. 7.

\section{Discussion}

The flagellar motor has a distinctive torque-speed relationship. At room temperature, torque is maximum at stall, it falls $\sim 10 \%$ between 0 and $\sim 160 \mathrm{~Hz}$, and then, as just noted, it drops rapidly, reaching 0 at $\sim 330 \mathrm{~Hz}^{15}$. In the high-torque, low-speed regime, torque is independent of temperature, and solvent isotope effects are relatively small; in the low-torque, high-speed regime, torque increases with temperature, and solvent isotope effects are large ${ }^{17}$. Evidently, at low speeds, rates of displacement of internal mechanical components or of translocation of protons are not limiting, whereas at high speeds, they are limiting. Comparison of our earlier results ${ }^{13}$ with those obtained here show that the switching behavior also is different in these two regimes, Fig. 8. In the high-torque, low-speed regime, the switching rates increase with speed, the $\mathrm{CW} \rightarrow \mathrm{CCW}$ rates more rapidly than the $\mathrm{CCW} \rightarrow \mathrm{CW}$ rates, thus increasing the $\mathrm{CCW}$ motor bias. In the low-torque, high-speed regime, the switching rates decrease with speed to an equivalent degree, without changing the motor bias. In the discussion that follows, we will attempt to understand the behavior near zero torque in terms of the mechanism for switching proposed by Duke et al. ${ }^{11}$. We do not yet understand the behavior at high torques.

Switching is commonly described by a thermal isomerization model, in which CW and CCW states are separated by an energy barrier ${ }^{18}$. The switching rates between these states are proportional to $\exp \left(-\Delta G^{\ddagger} / k T\right)$, where the activation energy $\Delta G^{\ddagger}$ is the difference between the free energy of the barrier and that of either the CW or CCW state. An increase in CheY-P binding lowers the free energy of the $\mathrm{CW}$ state and raises the free energy of the $\mathrm{CCW}$ state $^{12}$, so that the $\mathrm{CW} \rightarrow \mathrm{CCW}$ and $\mathrm{CCW} \rightarrow \mathrm{CW}$ switching rates change in a reciprocal manner. According to our measurements near zero torque, both switching rates $\left(\mathrm{k}_{\mathrm{CW}} \rightarrow \mathrm{CCW}\right.$ and $\mathrm{k}_{\mathrm{CCW} \rightarrow \mathrm{CW}}$ ) increase with torque by the same factor (a factor of about 4 for torques from 0 to $1300 \mathrm{pN} \mathrm{nm}$ ). This suggests a refinement in which the activation energies of the CW and CCW states are lowered by a stator-rotor interaction. If the magnitude of this change in free energy were of order $\tau \theta$, where $\tau$ is the motor torque and $\theta$ is the angular change in orientation of FliG (in a rotor-fixed coordinate system) that occurs when the motor switches, then one would expect an exponential increase in switching rate with torque, as predicted by a recent model of switching dynamics ${ }^{19}$. Instead, we observed switching rates that were linear with torque, so we sought a different explanation.

Consider the allosteric model proposed by Duke et al. ${ }^{11}$, in which a switching event is mediated by conformational changes in a ring of subunits that spread from subunit to subunit via nearestneighbor interactions. Each subunit exists in either an inactive or active state, states that promote $\mathrm{CCW}$ or $\mathrm{CW}$ rotation, respectively. The free energy of the active state relative to that of the inactive state changes from $+\mathrm{E}_{\mathrm{A}}$ to $-\mathrm{E}_{\mathrm{A}}$ when a subunit binds ligand (CheY-P); ligand binding favors the active state. The coupling energy between adjacent subunits is $-\mathrm{E}_{\mathrm{J}}$ if they are in the same state but zero otherwise; this favors like conformations. Switching from the fully inactive $(\mathrm{CCW})$ to the fully active $(\mathrm{CW})$ configuration occurs in two steps. First, a subunit undergoes a conformational change to nucleate an active domain. Then, this domain grows until it spreads throughout the entire ring. The nucleation occurs at a rate $N \omega \exp (-\Delta E / k T)$, where $N=34$ is the number of subunits in the ring, $\omega$ is the rate of conformational transition in either direction for each subunit, and $\Delta E=2 E_{\mathrm{J}}-E_{\mathrm{A}}\left(\right.$ or $\left.2 E_{\mathrm{J}}+E_{\mathrm{A}}\right)$ depending upon whether or not a ligand is bound to the subunit ${ }^{11}$. The probability that the domain grows from size 1 to size

$N$ without shrinking to zero is limited by the ligand binding rate, $k_{\text {ligand. }}$. Because of different binding constants for active and inactive subunits, an active domain nucleated by chance will 
remain energetically disfavored until it is stabilized by ligand binding. If the subunit transition frequency is increased by a factor $\exp (\tau \theta / k T)$, the active subunit will have less time to wait for ligand binding before it transits back to the inactive conformation, thereby decreasing the probability that the nucleation event leads to switching. Therefore, the effect of the stator-rotor interaction on rates of switching will be less than exponential.

To determine the exact dependence of switching rates on motor torque, we performed MonteCarlo simulations of the Duke et al. model, and the results are shown in Fig. 9 A and B. The switching rates increased nearly linearly with torque before reaching a plateau, where the plateau was due to the limitation of the ligand binding rate. To obtain a factor of about 4 increase before reaching the plateau, we set the value of $k_{\text {ligand }}$ to be $30 \mathrm{~s}^{-1}$, larger than assumed in ref. $11\left(10 \mathrm{~s}^{-1}\right)$, but close to the value measured in ref. ${ }^{6}\left(\sim 20 \mathrm{~s}^{-1}\right)$. All other parameters were similar to those of ref. ${ }^{11}: E_{A}=1 k T, E_{J}=4 k T, \omega=0.5 \times 10^{4}$ and $E_{\mathrm{L}}$ (the decrease in free energy of a subunit upon binding the ligand CheY-P $)=-0.085 k T$, giving the wild-type $\mathrm{CCW}$ bias of about $80 \%$. We fit the simulation curves (Fig. 9 A, B) to our data using two free parameters, $\theta$ and a scaling factor for the vertical axis (for the switching rates). We could fit both sets of data with $\theta / k T=3.3 \times 10^{-3}(\mathrm{pN} \mathrm{nm})^{-1}$, corresponding to $\theta=0.8^{\circ}$ (Fig. $9 \mathrm{C}, \mathrm{D}$ ). Thus, our results are consistent with the allosteric model proposed by Duke et al. We note that there must be variations in the orientations of different FliG molecules depending upon stator-rotor interactions, so that $0.8^{\circ}$ is some appropriately-weighted average value, with $\tau \theta$ the average amount of work performed by the stator on the rotor to promote switching.

\section{Materials and Methods}

\section{Bacteria and cultures}

E. coli strain KAF83 was used to monitor wild-type motors. It is a derivative of AW40520 carrying null mutations in pilA and fliC, but otherwise wild type for motility and chemotaxis. Cells were grown at $33^{\circ} \mathrm{C}$ in T-broth $(1 \%$ tryptone, $0.5 \% \mathrm{NaCl})$ supplemented with the appropriate antibiotics to $\mathrm{OD}_{600} \sim 0.5$.

\section{Sample preparation}

Cells were washed twice by centrifugation at $3000 \times \mathrm{g}$, and resuspended in motility medium (10 mM potassium phosphate, $0.1 \mathrm{mM}$ EDTA, $10 \mathrm{mM}$ lactate, $70 \mathrm{mM} \mathrm{NaCl}, \mathrm{pH} 7.0$ ). Gold spheres of diameter 100, 150, or $200 \mathrm{~nm}(15711,15712,15713$, Ted Pella Inc.) were attached to the hooks of KAF83 cells with anti-hook antibody, following the procedure described in ref. 14. A sample was placed on a glass coverslip coated with poly-L-lysine (P4707, Sigma) and allowed to stand for $5 \mathrm{~min}$. The coverslip was installed as the top window of a flow cell ${ }^{21}$ and rinsed with motility medium. A 30\% w/v stock solution of Ficoll 400 (dialyzed and lyophilized, 46324 , Sigma) was prepared in motility medium, and other concentrations $(5 \%, 10 \%$, and $15 \%$ w/v) were made from this stock solution by dilution with motility medium.

\section{Laser dark-field microscopy}

The apparatus is shown in Fig. 1. It was modified from that of ref. ${ }^{14}$ by addition of a 50/50 beam splitter, two $3 \times 1 \mathrm{~mm}$ precision slits (NT39-908, Edmund Optics) mounted at right angles to one another, and a second photomultiplier tube (identical to the first). Slit 1 gave the $x$ position information, while slit2 gave the $y$ information. When the center of rotation of the image of a gold sphere was aligned at one of the corners of the intersection of the slits, the phases of the signals from the two photomultiplier tubes differed by 90 or -90 degrees, depending upon the direction of rotation, as shown in Fig. 2. 


\section{Experimental procedure}

The microscope stage was moved to align the center of rotation of the image of a gold sphere to a corner of the slit intersection, as described above. Data were accumulated for a period of $3 \mathrm{~min}$ for each motor, for 3 different sizes of gold spheres, 100, 150, and $200 \mathrm{~nm}$ in $0 \%$ Ficoll, and for $200 \mathrm{~nm}$ gold spheres in 3 different concentrations of Ficoll, 5\%, 10\% and 15\%.

Solutions were exchanged (drawn through the flow cell) at a rate of $70 \mu \mathrm{l} / \mathrm{min}$. When working with Ficoll, 7 min was allowed to ensure that the flow cell reached equilibrium. In all cases, the flow was continued throughout the experiment. All experiments were performed at room temperature $\left(23^{\circ} \mathrm{C}\right)$.

\section{Data analysis}

The anode currents from the two photomultiplier tubes were converted to voltages, which were DC-coupled to two 8-pole low-pass Bessel filters (3384, Krohn-Hite) with a cut-off frequency of $700 \mathrm{~Hz}$. The filter outputs were sampled at $3 \mathrm{KHz}$ by a computer data-acquisition system controlled by LabVIEW (National Instruments, Austin, TX). Offline data analysis was done by using custom scripts in MATLAB (MathWorks, Natick, MA). The $x$ and $y$ signals (approximating cosine and sine functions) were scaled so that the peak values ranged from -1 to +1 , and then converted to polar coordinates. The angle was differentiated with respect to time to yield the rotation rate (CW positive and CCW negative), and the output was smoothed with a 40-point running average. Switching was determined with a two-threshold-crossing algorithm: for each 3-min measurement, the speed histogram was plotted, and the peak positions at positive and negative velocities were extracted, corresponding to the steady-state $\mathrm{CW}$ and $\mathrm{CCW}$ rotation rates, $\mathrm{v}^{+}$and $\mathrm{v}^{-}$. The span between these values, $\left(\mathrm{v}^{+}-\mathrm{v}^{-}\right)$, was divided by three to give three intervals. Switching from $\mathrm{CW}$ to $\mathrm{CCW}$ was flagged when the velocity crossed from the top interval to the bottom interval, and switching from CCW to CW was flagged when the velocity crossed from the bottom interval to the top interval. The time resolution was about $10 \mathrm{~ms}$, limited mostly by the 40-point running average.

Torque was calculated from the rotational frictional drag coefficient multiplied by the angular velocity. For a gold sphere on a hook, neglecting the contribution of the hook, the drag coefficient is $8 \pi \eta a^{3}+6 \pi \eta a l^{2}$, where $a$ is the radius of the sphere, $\eta$ is the viscosity of the medium, and $l$ is the rotational eccentricity, i.e., the distance between the rotational axis and the center of the sphere. The drag coefficient ranges from $8 \pi \eta a^{3}$ to $14 \pi \eta a^{3}$ for $l$ ranging from 0 to $a$. The average value $11 \pi \eta a^{3}$ was chosen with an estimated error of $20 \%$. The values of viscosity for various concentrations of Ficoll were obtained from ref. ${ }^{15}$. For a latex bead on a filament stub, i.e., for the data from ref. ${ }^{13}$, the drag is the sum of contributions from the rotation of the bead and the filament stub. Calculation of the drag coefficient in this case was done as in refs. ${ }^{22}$;

${ }^{23}$, assuming a bead rotational eccentricity of $0.2 \mu \mathrm{m}$ and a filament stub length of $1 \mu \mathrm{m}$. The error was estimated to be $10 \%$, resulting from uncertainties in eccentricity, stub length, and bead radius.

\section{Acknowledgments}

We thank T.S. Shimizu and Y. Tu for helpful discussions. This work was supported by National Institutes of Health Grant AI016478.

\section{References}

1. Turner L, Ryu W, Berg HC. Real-time imaging of fluorescent flagellar filaments. J Bacteriol 2000;182:2793-2801. [PubMed: 10781548]

2. Berg HC, Brown DA. Chemotaxis in Escherichia coli analysed by three-dimensional tracking. Nature 1972;239:500-504. [PubMed: 4563019] 
3. Kojima S, Blair DF. Conformational change in the stator of the bacterial flagellar motor. Biochemistry 2001;40:13041-13050. [PubMed: 11669642]

4. Blair DF. movement driven by proton translocation. FEBS Lett 2003;545:86-95. [PubMed: 12788496]

5. Cluzel P, Surette M, Leibler S. An ultrasensitive bacterial motor revealed by monitoring signaling proteins in single cells. Science 2000;287:1652-1655. [PubMed: 10698740]

6. Sourjik V, Berg HC. Binding of the Escherichia coli response regulator CheY to its target measured in vivo by fluorescence resonance energy transfer. Proc Natl Acad Sci USA 2002;99:12669-12674. [PubMed: 12232047]

7. Welch M, Oosawa K, Aizawa SI, Eisenbach M. Phosphorylation-dependent binding of a signal molecule to the flagellar switch of bacteria. Proc Natl Acad Sci USA 1993;90:8787-8791. [PubMed: 8415608]

8. Bai F, Lo CJ, Berry RM, Xing J. Model studies of the dynamics of bacterial flagellar motors. Biophys J 2009;96:3154-3167. [PubMed: 19383460]

9. Meacci G, Tu Y. Dynamics of the bacterial flagellar motor with multiple stators. Proc Natl Acad Sci USA 2009;106:3746-3751. [PubMed: 19234112]

10. Alon U, Camarena L, Surette MG, Aguera y Arcas B, Liu Y, Leibler S, Stock JB. Response regulator output in bacterial chemotaxis. EMBO J 1998;17:4238-4248. [PubMed: 9687492]

11. Duke TAJ, Le Novère N, Bray D. Conformational spread in a ring of proteins: a stochastic approach to allostery. J Mol Biol 2001;308:541-553. [PubMed: 11327786]

12. Sharf BE, Fahrner KA, Turner L, Berg HC. Control of direction of flagellar rotation in bacterial chemotaxis. Proc Natl Acad Sci USA 1998;95:201-206. [PubMed: 9419353]

13. Fahrner KA, Ryu WS, Berg HC. Bacterial flagellar switching under load. Nature 2003;423:938. [PubMed: 12827190]

14. Yuan J, Berg HC. Resurrection of the flagellar motor near zero load. Proc Natl Acad Sci USA 2008;105:1182-1185. [PubMed: 18202173]

15. Chen X, Berg HC. Torque-speed relationship of the flagellar rotary motor of Escherichia coli. Biophys J 2000a;78:1036-1041. [PubMed: 10653817]

16. Berg HC, Turner L. Torque generated by the flagellar motor of Escherichia coli. Biophys J 1993;65:2201-2216. [PubMed: 8298044]

17. Chen X, Berg HC. Solvent-isotope and $\mathrm{pH}$ effects on flagellar rotation in Escherichia coli. Biophys J 2000b;78:2280-2284. [PubMed: 10777726]

18. Khan S, Macnab RM. The steady-state counterclockwise/clockwise ratio of bacterial flagellar motors is regulated by protonmotive force. J Mol Biol 1980;138:563-597. [PubMed: 6774099]

19. Van Albada, SB.; Tanase-Nicola, S.; ten Wolde, PR. 2008. The switching dynamics of the bacterial flagellar motor. arXiv:0811.2437v1

20. Armstrong JB, Adler J, Dahl MM. Nonchemotactic mutants of Escherichia coli. J Bacteriol 1967;93:390-398. [PubMed: 5335897]

21. Berg HC, Block SM. A miniature flow cell designed for rapid exchange of media under high-power microscope objectives. J Gen Microbiol 1984;130:2915-2920. [PubMed: 6396378]

22. Ryu WS, Berry RM, Berg HC. Torque-generating units of the flagellar motor of Escherichia coli have a high duty ratio. Nature 2000;403:444-447. [PubMed: 10667798]

23. Reid SW, Leake MC, Chandler JH, Lo C, Armitage JP, Berry RM. The maximum number of torquegenerating units in the flagellar motor of Escherichia coli is at least 11. Proc Natl Acad Sci USA 2006;103:8066-8071. [PubMed: 16698936] 


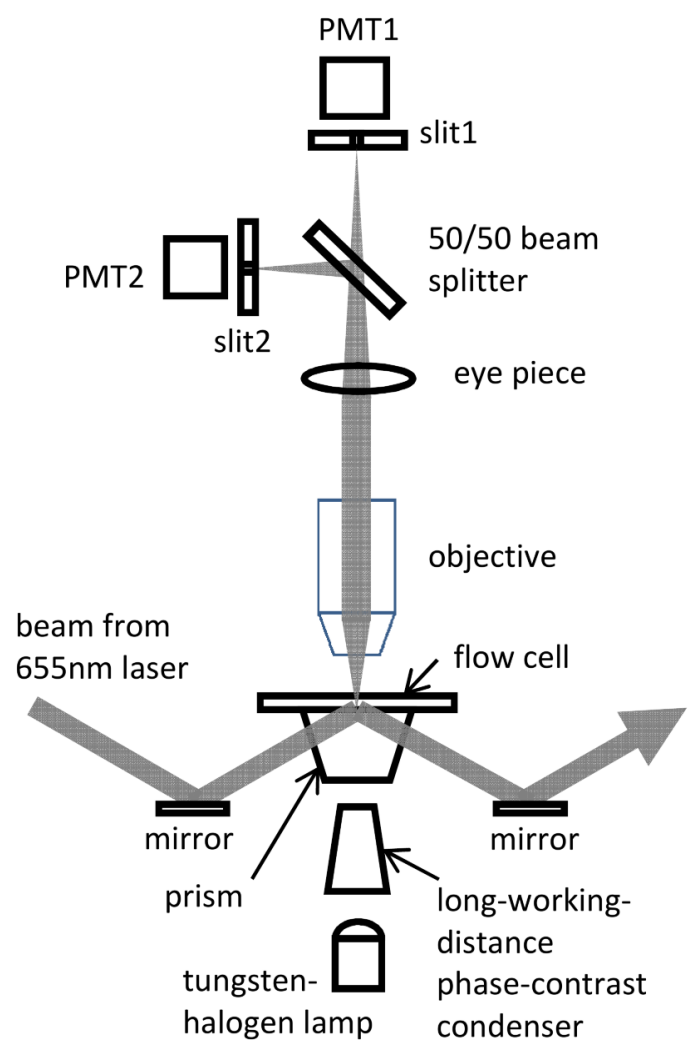

Fig. 1.

Schematic view of the apparatus: $655 \mathrm{~nm}$ laser light scattered by a gold sphere was sampled by a 50/50 beam splitter, focused onto two $3 \times 1 \mathrm{~mm}$ slits mounted at right angles to one another, and collected by a photomultiplier tube (PMT) behind each slit. The truncated $60^{\circ}$ prism and the long-working-distance phase-contrast condenser allowed cells to be observed by phase contrast. 

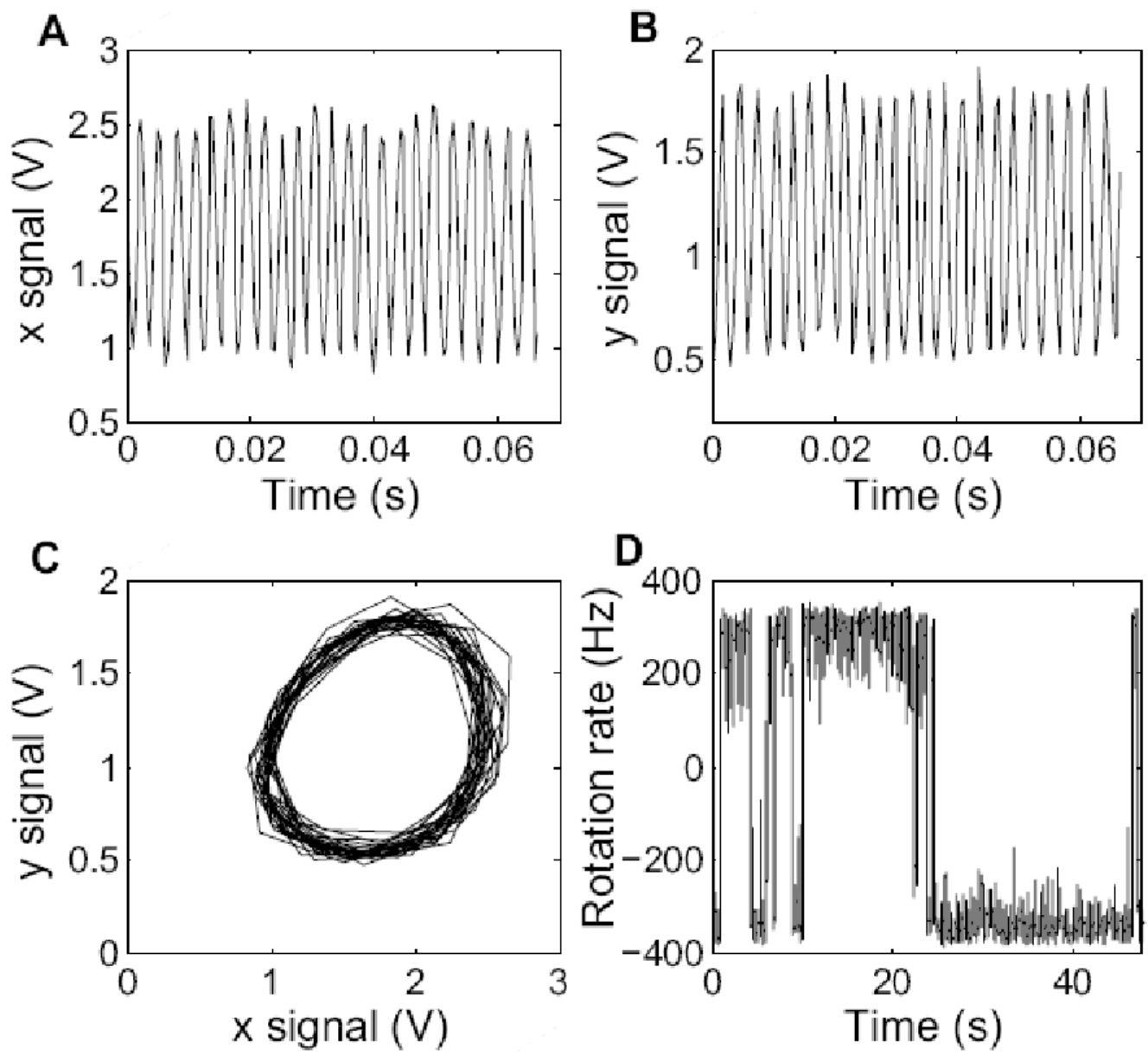

Fig. 2.

(A) $x$ signal (PMT1) as a function of time. (B) $y$ signal (PMT2) as a function of time. (C) a plot of $y$ versus $x$, showing the trajectory of the image of the gold sphere. (D) a sample trace showing switching: rotation rate of the sphere as a function of time. '-': counter-clockwise. '+': clockwise. The signals are voltages proportional to the PMT output currents. 

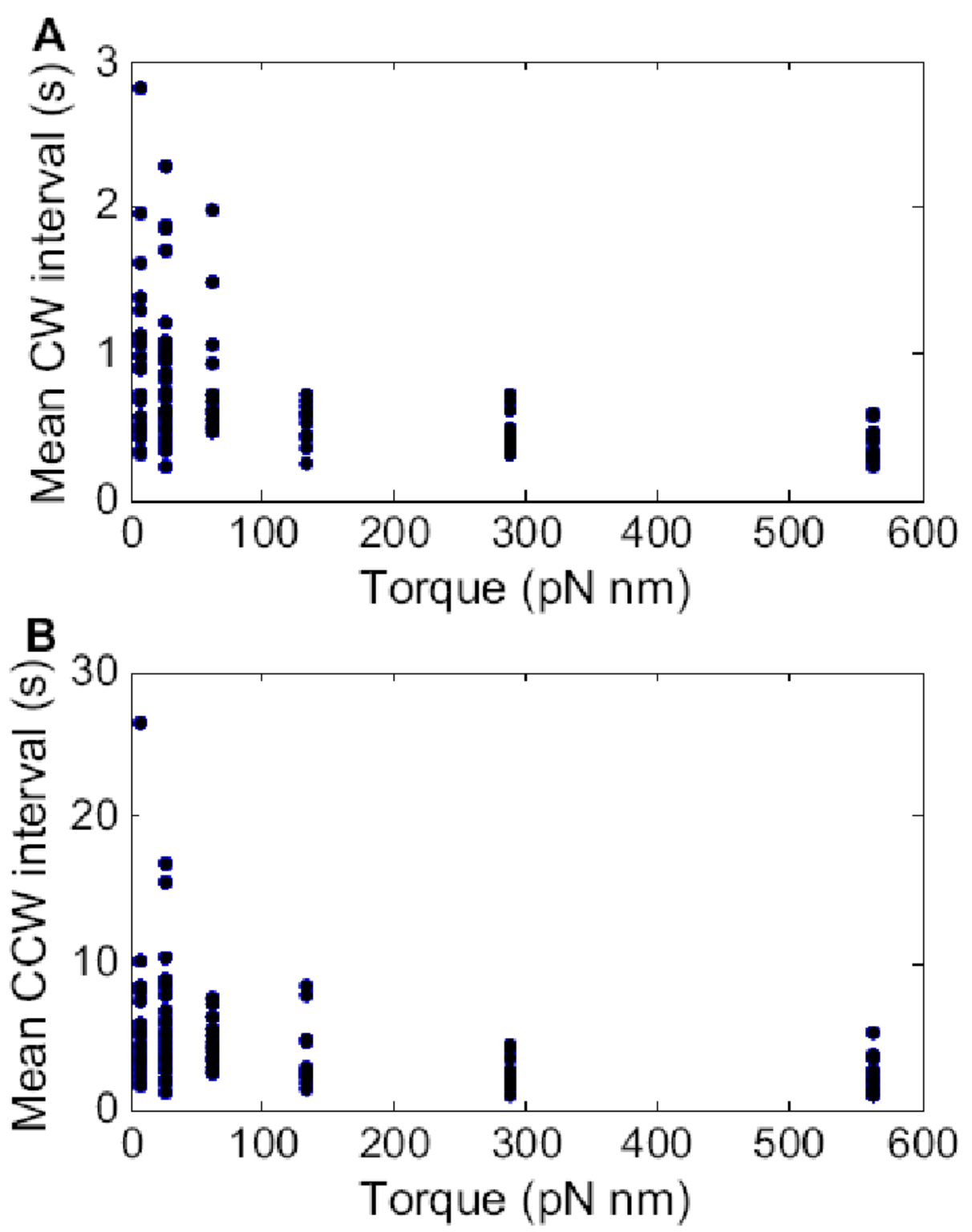

Fig. 3.

(A) Mean CW intervals, and (B) mean CCW intervals, plotted as a function of motor torque. The symbols are values for each cell. Reading from left to right, the load conditions were 100, 150 or 200-nm diameter gold spheres in motility medium, and 200-nm diameter gold spheres in $5 \%, 10 \%$, or $15 \%$ Ficoll. The number of cells studied at each of these load conditions were $29,45,19,15,15$, and 15, respectively. 

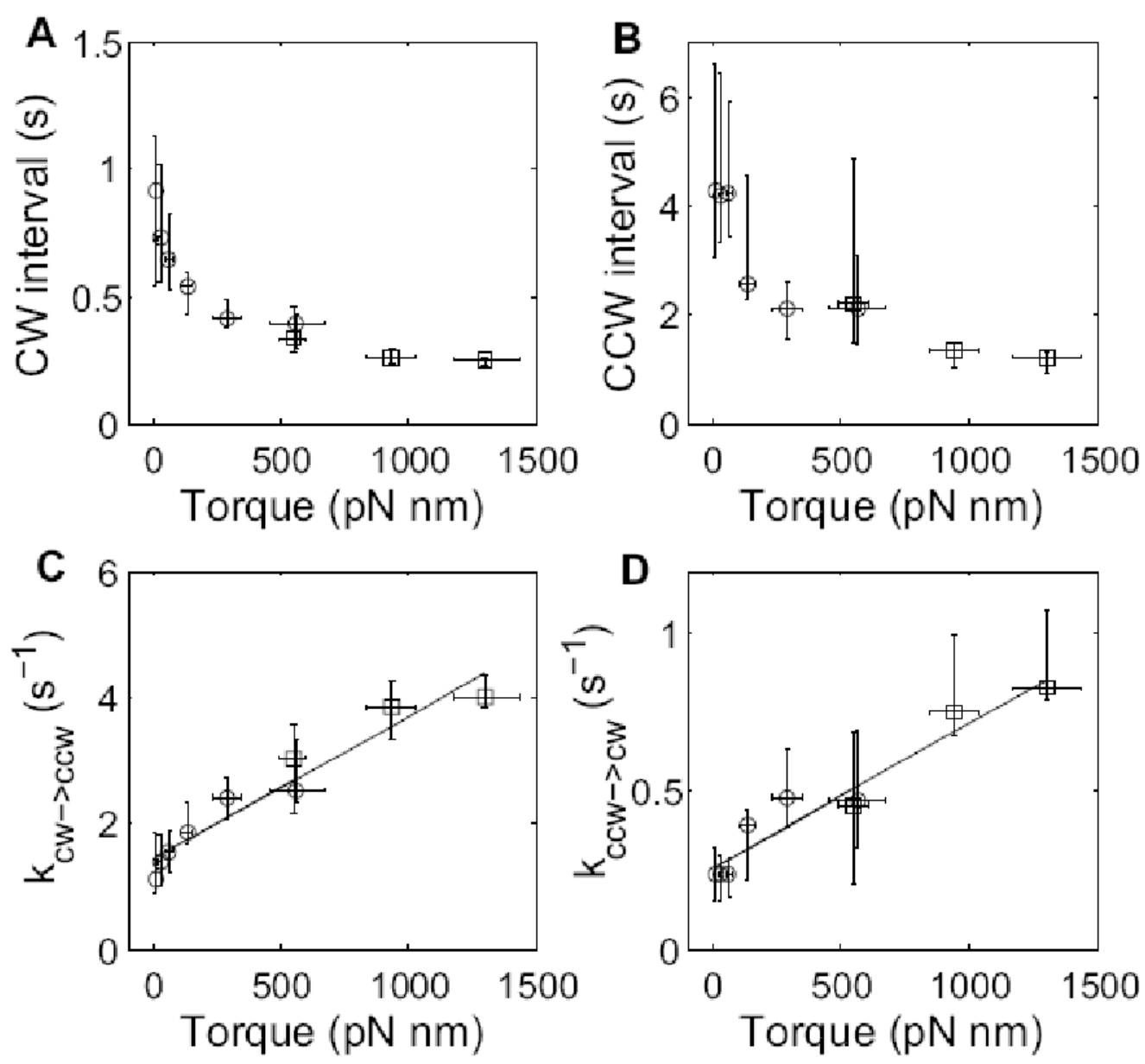

Fig. 4.

Median and interquartile range for (A) CW intervals and (B) CCW intervals, plotted as a function of motor torque. The corresponding rate constants $\mathrm{k}_{\mathrm{CW} \rightarrow \mathrm{CCW}}=1 /\langle\mathrm{CW}\rangle$ and $\mathrm{k}_{\mathrm{CCW} \rightarrow \mathrm{CW}}=1 /<\mathrm{CCW}>$ are plotted in $(\mathbf{C})$ and (D), along with linear fits. Open circles are results from this study, while open squares are results from ref. ${ }^{13}$. 


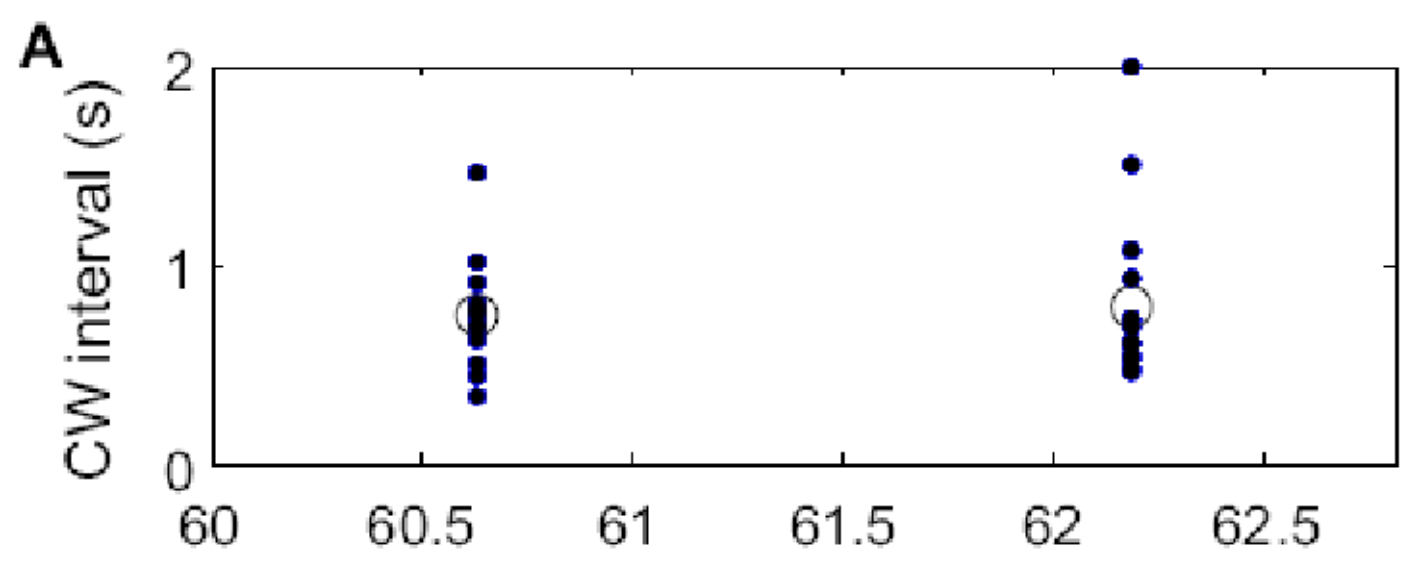

B
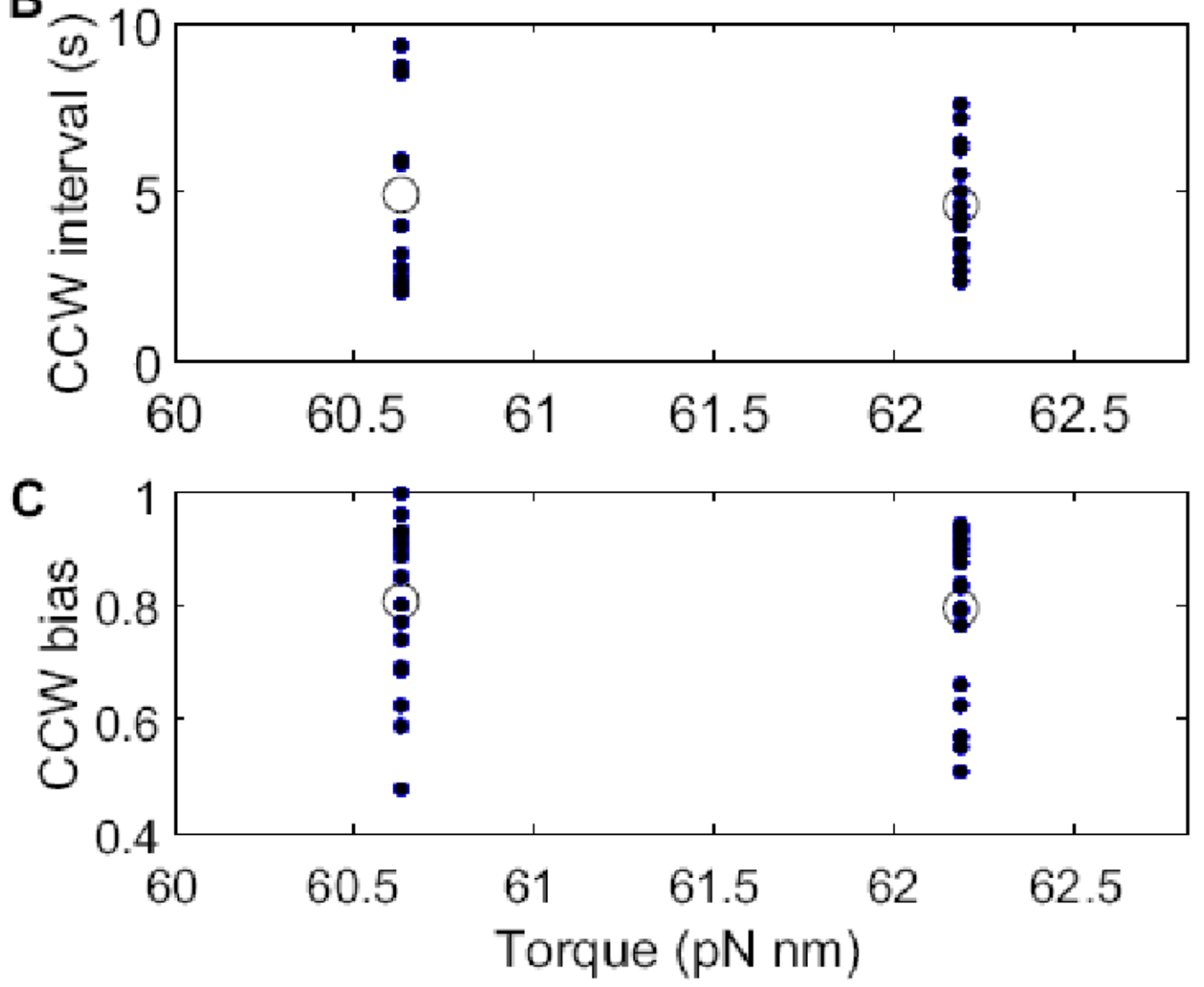

Fig. 5.

(A) Mean CW intervals, (B) mean CCW intervals, and (C) CCW biases, under two conditions with equivalent loads: (left) gold spheres of diameter $150 \mathrm{~nm}$ in $5 \%$ Ficoll; (right) gold spheres of diameter $200 \mathrm{~nm}$ in motility medium. Note that the motor torques in these two cases were nearly the same: the horizontal scale is expanded. The closed dots are values for each cell, and the open circles are means for each load condition. The number of cells studied on the left was 17 , and on the right 15 . 

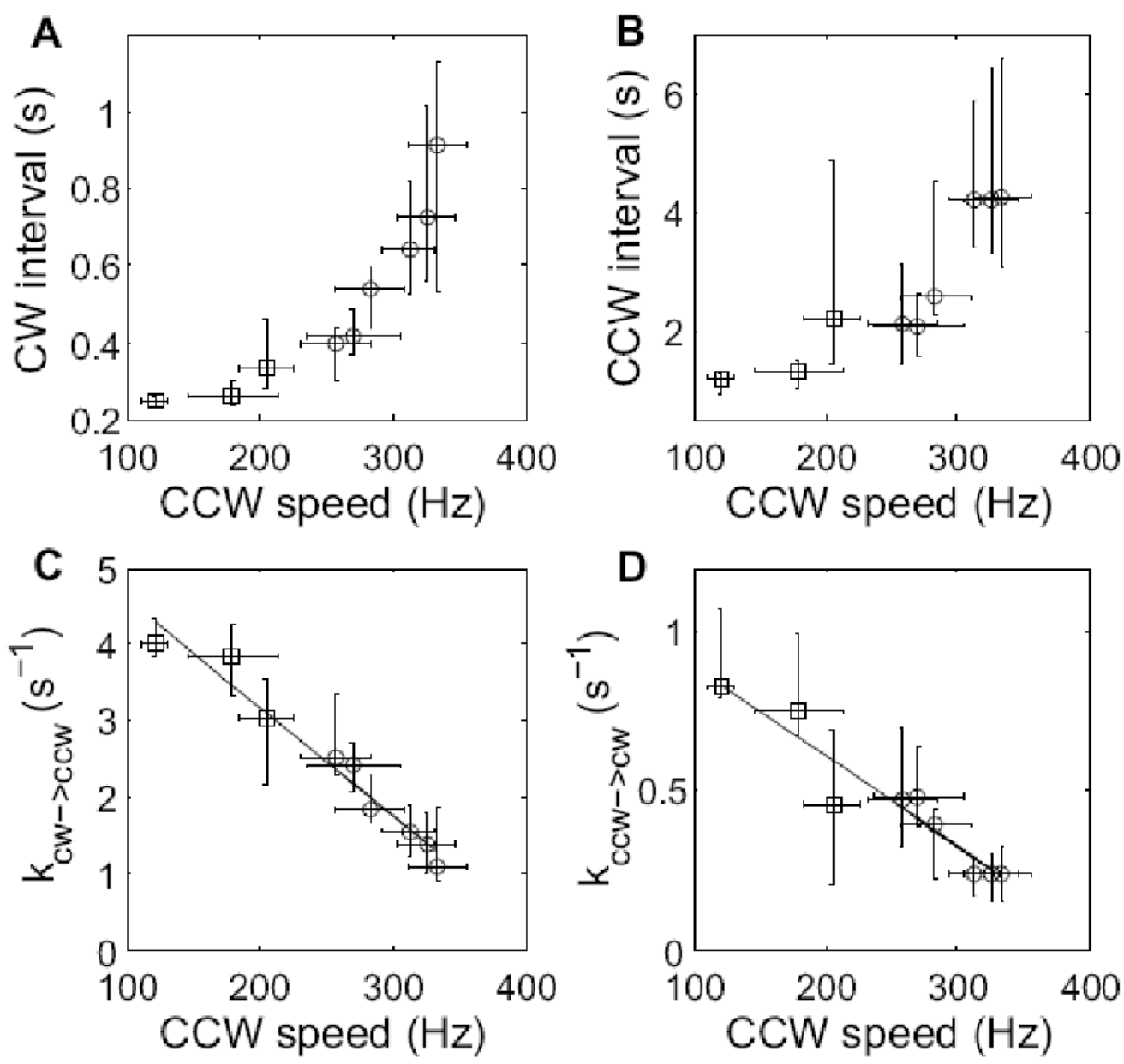

Fig. 6.

Median and interquartile range for (A) $\mathrm{CW}$ intervals and (B) $\mathrm{CCW}$ intervals, plotted as a function of $\mathrm{CCW}$ speed. The corresponding rate constants $\mathrm{k}_{\mathrm{CW} \rightarrow \mathrm{CCW}}=1 /\langle\mathrm{CW}\rangle$ and $\mathrm{k}_{\mathrm{CCW} \rightarrow \mathrm{CW}}=1 /<\mathrm{CCW}>$ are plotted in (C) and (D), along with linear fits. Open circles are results from this study, while open squares are results from ref. ${ }^{13}$. 


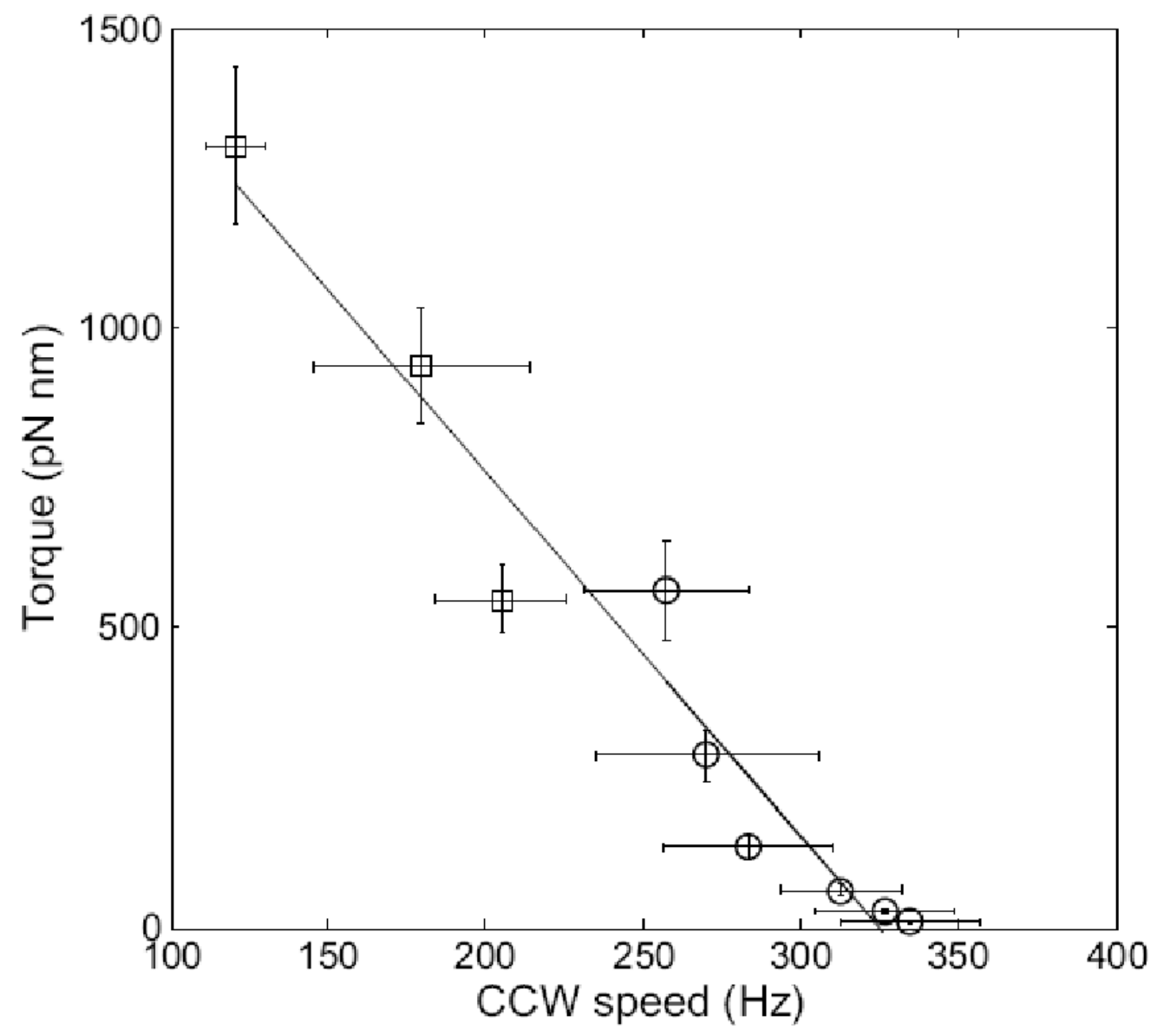

Fig. 7.

Mean torque as a function of mean CCW speed, along with a linear fit. Open circles are results from this study, while open squares are results from ref. ${ }^{13}$. 

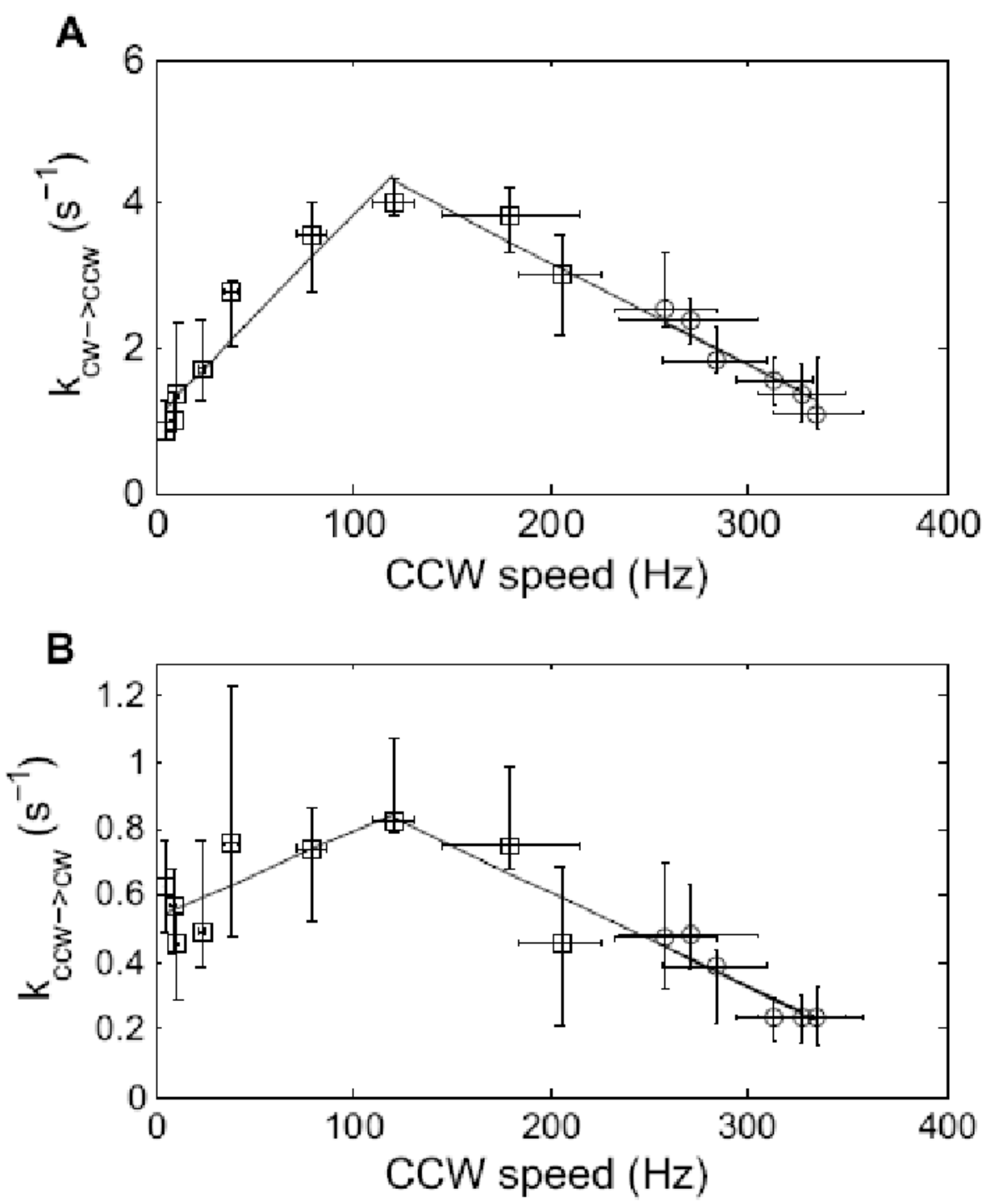

Fig. 8.

The rate constants $\mathrm{k}_{\mathrm{CW} \rightarrow \mathrm{CCW}}=1 /\langle\mathrm{CW}\rangle$ and $\mathrm{k}_{\mathrm{CCW} \rightarrow \mathrm{CW}}=1 /\langle\mathrm{CCW}\rangle$ plotted as a function of $\mathrm{CCW}$ speed, along with linear fits. Note the extension of the speed axis to $0 \mathrm{~Hz}$. Open circles are results from this study, while open squares are results from ref. ${ }^{13}$. 

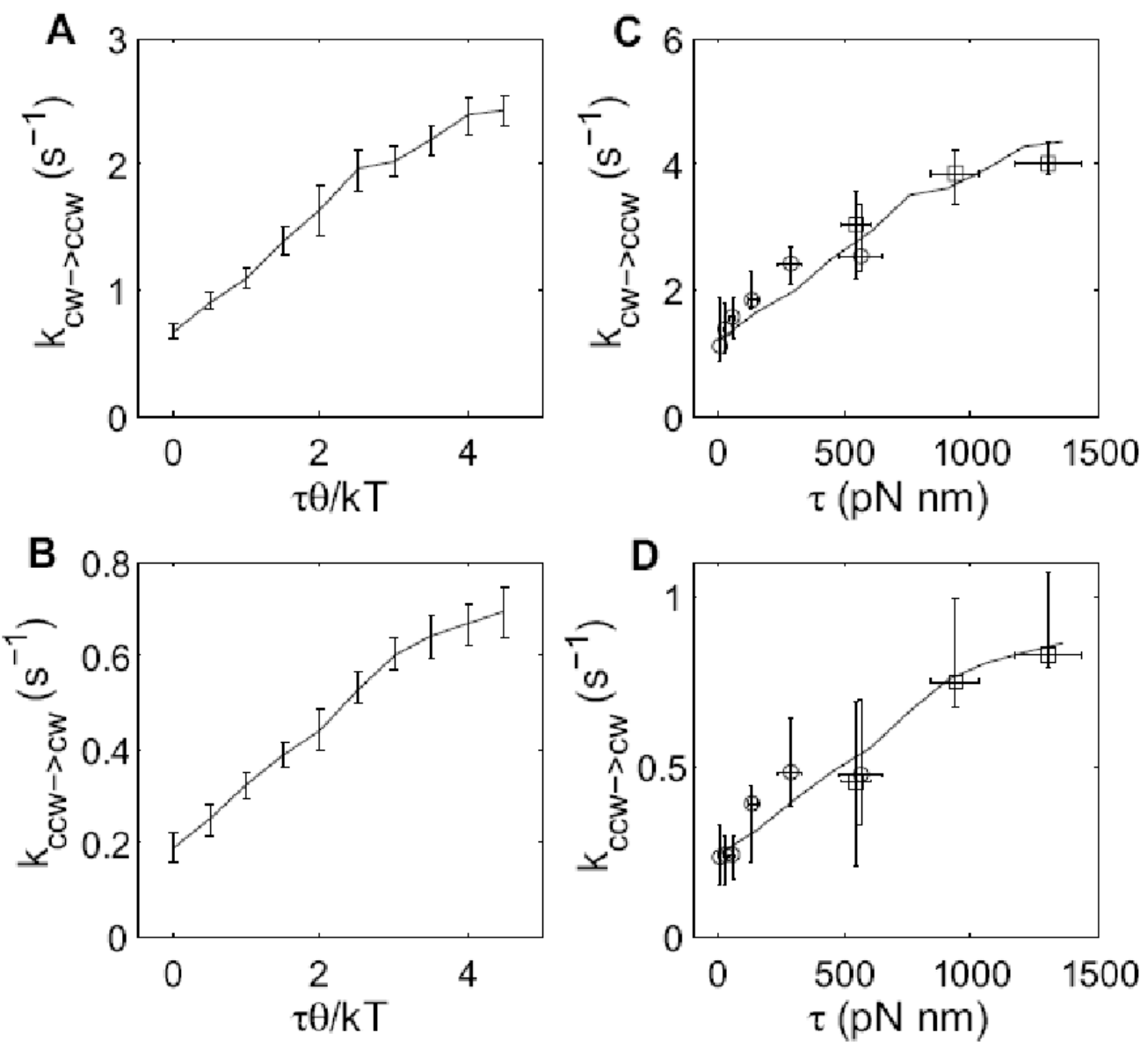

Fig. 9.

$(\mathbf{A}, \mathbf{B})$ Monte-Carlo simulations of the model of Duke et al. ${ }^{11}$. Each data point is the mean and standard deviation of 8 independent simulations, and each simulation is a $500 \mathrm{~s}$ long switching trace. $(\mathbf{C}, \mathbf{D})$ Fitting of the simulation to the measurements, with two free parameters in each fit: $\theta$ and a scaling factor in the vertical axis. Symbols with error bars are the measurements, and lines are the fitting of simulations. 